\title{
MELHORIA DO FATOR DE POTÊNCIA ATRAVÉS DO AUMENTO DO NÚMERO DE PULSOS DE CONVERSORES GRAETZ COMUTADOS PELA REDE: MODELAGEM E RESULTADOS EXPERIMENTAIS
}

\author{
Angelo J. J. Rezek; José P. G.de Abreu; Valberto F.da Silva; José M. E. Vicente; José A. Cortez; \\ Otavio H. S. Vicentini; Adriana Cortez de Sá; Adriana A. S. Izidoro \\ Instituto de Engenharia Elétrica - UNIFEI - Universidade Federal de Itajubá \\ C.P.: 50 CEP:37500-903 Itajubá-MG - Brasil \\ e-mail: rezek@iee.efei.br \\ Mauro S. Miskulin \\ FEEC/UNICAMP - Universidade Estadual de Campinas \\ C.P.: 6101 CEP:13081-970 Campinas-SP - Brasil
}

Resumo - A corrente alternada (CA) de conversores comutados pela rede é não senoidal, gerando desta maneira, harmônicos em sistemas elétricos.

Conversores Graetz de seis pulsos são amplamente utilizados na indústria. Conversores de doze pulsos podem ser implementados a partir da associação de dois conversores de seis pulsos em série ou em paralelo. No entanto, é necessária a utilização de transformadores de alimentação. Estes transformadores devem ser conectados de modo que seus ângulos de tensão de fase sejam defasados de trinta graus entre si. Harmônicos de ordem inferior $\left(5^{\circ}\right.$ e $\left.7^{\circ}\right)$ são eliminados neste caso, tornando estes transformadores adequados para a aplicação proposta.

Uma excelente redução de harmônicos pode ser obtida quando multiconversores de 24 e 48 pulsos são implementados, pela associação de quatro ou oito conversores graetz de seis pulsos, respectivamente. Quando o número de pulsos do conversor é aumentado, haverá também uma melhoria no fator de potência do sistema. Resultados experimentais serão apresentados e discutidos.

Abstract - The alternating current (AC) of linecommutated converters is non-sinusoidal, generating, therefore, harmonics in the electric system.

Six-pulse graetz line-commutated converters are widely employed in industry. Twelve-pulse converter can be achieved by associating two six-pulse converters in series or in parallel. However it is necessary to use supply transformers. These transformers are connected in such a way that the secondary voltage phase angles are displaced thirty degrees in relation to each other. Lower order characteristic harmonics are eliminated in this case $\left(5^{\circ}, 7^{\circ}\right)$, rendering these transformers as adequate for the proposed application.

A very good harmonic mitigation can be obtained when 24 or 48 - pulse multiconverters are employed, by associating four or eight graetz six - pulse converters, respectively. When the converter pulse number is increased there will be also, a system power factor improvement. Experimental results will be presented and discussed.

\section{NOMENCLATURA}

$\mathrm{Fp} \Rightarrow$ Fator de potência do conversor.

$\mathrm{I}_{1} \Rightarrow$ Valor (RMS) da corrente fundamental de fase.

$\mathrm{I} \Rightarrow$ Valor (RMS) da corrente de fase.

$\phi_{1} \Rightarrow$ Ângulo de fase da corrente fundamental $\mathrm{I}_{1}$.

$\mu \Rightarrow$ Ângulo de comutação (em radianos).

$\alpha \Rightarrow$ Ângulo de disparo do conversor.

$\delta \Rightarrow$ Ângulo auxiliar.

$\mathrm{I}_{\mathrm{d}} \Rightarrow$ Corrente no lado CC (corrente contínua).

$\mathrm{m} \Rightarrow$ Número de pontes conectadas em série.

$\mathrm{I}_{\mathrm{n}} \Rightarrow$ Valor (RMS) do harmônico de corrente de ordem $\mathrm{n}$.

$\mathrm{n} \Rightarrow$ Ordem do harmônico.

$\mathrm{I}_{\mathrm{n} 0} \Rightarrow$ Valor (RMS) da corrente harmônica de ordem n, para ângulo de comutação $\mu=0^{\circ}$.

$\mathrm{I}_{10} \Rightarrow$ Valor (RMS) da corrente fundamental, para ângulo de comutação $\mu=0^{\circ}$.

$\mathrm{DA} \Rightarrow$ Defasagem Angular.

FRH $\Rightarrow$ Fator de redução de harmônicos.

$\mathrm{FDH} \Rightarrow$ Fator de distorção de harmônicos.

$\mathrm{H} ; \mathrm{K} ; \mathrm{H}_{1} ; \mathrm{K}_{1} \Rightarrow$ Valores auxiliares para cálculo de FRH.

$\mathrm{k} \Rightarrow$ Variável auxiliar para determinação de $\mathrm{n}(\mathrm{k}=0,1,2,3 \ldots)$.

\section{I - INTRODUÇÃO}

Transformadores especiais conectados em delta zig-zag ($\left.15^{\circ}, 0^{\circ}, 15^{\circ}, 30^{\circ}\right)$ ou estrela - delta estendido $\pm\left(15^{\circ}\right)$ podem ser utilizados para que se obtenha $15^{\circ}$ de defasamento para implementação de multiconversores de 24 pulsos. No caso de um multiconversor de 48 pulsos, são necessários $7,5^{\circ}$ de defasamento angular na tensão secundária, o qual pode ser obtido através da utilização dos transformadores especiais mencionados anteriormente. Um autotransformador especial $\mathrm{ADZ}\left(7,5^{\circ}\right)$ [10] foi também desenvolvido e utilizado, como mostra a figura 9.

Uma recente e importante aplicação de sistemas multiconversores pode ser exemplificada na utilização do 48-pulse Static Var Generator - SVG (gerador estático de reativos de 48 pulsos), no sistema apresentado em [16] e em inversores de 24 pulsos para serem aplicados em Adjusted Speed Drives - ASD (Acionamentos de velocidade regulável). Estes ASD's, oferecem significativas vantagens 
em aplicações de processos de ventilação e bombeamento, em termos de alta eficiência e performance, apresentando maior confiabilidade em processos de áreas críticas, como por exemplo, bombeamento de petróleo [17].

\section{II - FATOR DE POTÊNCIA DE CONVERSORES}

O fator de potência de conversores pode ser obtido pela expressão:

$F p=\left(\frac{I_{1}}{I}\right) \cos \phi_{1}$

$\delta=\alpha+\mu$

Sendo $\delta$ um ângulo auxiliar igual à soma dos ângulos de disparo e de comutação.

Considerando-se o efeito da comutação, tem-se que [2]:

$\operatorname{tg} \phi_{1}=\frac{2 \mu+\operatorname{sen} 2 \alpha-\operatorname{sen} 2 \delta}{\cos 2 \alpha-\cos 2 \delta}$

Desprezando-se o efeito da comutação pode-se obter a seguinte expressão:

$\phi_{1}=\alpha$

Novamente desprezando-se o efeito da comutação [8]:

$I_{1}=\frac{\sqrt{6}}{\pi} \mathrm{I}_{\mathrm{d}} \mathrm{m}$

$I_{n}=\frac{I_{1}}{n}$

\section{III - REDUCÃO DE HARMÔNICOS ATRAVÉS DO EFEITO DA COMUTAÇÃO}

A comutação é um fator de redução de harmônicos. As expressões (7) a (9) mostram o cálculo do Fator de Redução de Harmônicos $\left(F R H=I_{n} / I_{n 0}\right)$ [7],[2].

$$
\begin{aligned}
& H=\left[\frac{\operatorname{sen}((n+1) \mu / 2)}{n+1}\right] \\
& K=\left[\frac{\operatorname{sen}((n-1) \mu / 2)}{n-1}\right] \\
& \frac{\mathrm{I}_{\mathrm{n}}}{\mathrm{I}_{\mathrm{n} 0}}=\frac{\sqrt{\mathrm{H}^{2}+\mathrm{K}^{2}-2 \mathrm{HK} \cos (2 \alpha+\mu)}}{(\cos \alpha-\cos \delta)}
\end{aligned}
$$
[2]:

A expressão (9) não é válida para $\mathrm{n}=1$ e de acordo com

$\mathrm{H}_{1}=\cos 2 \alpha-\cos 2 \delta$
$\mathrm{K}_{1}=\operatorname{sen} 2 \delta-\operatorname{sen} 2 \alpha-2 \mu$

$\frac{\mathrm{I}_{1}}{\mathrm{I}_{10}}=\frac{\sqrt{\mathrm{H}_{1}{ }^{2}+\mathrm{K}_{1}{ }^{2}}}{4(\cos \alpha-\cos \delta)}$

A Tabela 1 mostra o Fator de Redução de Harmônicos (FRH), considerando-se as expressões (9), (12) e também os resultados obtidos através da utilização de um programa FFT [5], para 256 amostras. Considerou-se para o conversor de doze pulsos que $\alpha=0^{\circ}$ e $\mu=15^{\circ}$.

TABELA 1

FRH para $\alpha=0^{\circ}$ e $\mu=15^{\circ}$.

\begin{tabular}{|c|c|c|}
\hline $\begin{array}{c}\mathrm{n} \\
\text { Ordem } \\
\text { Harmônica }\end{array}$ & $\begin{array}{c}\text { FRH } \\
\text { Programa } \\
\text { FFT }\end{array}$ & $\begin{array}{c}\text { FRH } \\
\text { Expressões (9) } \\
\text { e (12) }\end{array}$ \\
\hline 1 & 0,9981 & 0,9980 \\
\hline 11 & 0,7899 & 0,7899 \\
\hline 13 & 0,7175 & 0,7174 \\
\hline 23 & 0,3457 & 0,3453 \\
\hline 25 & 0,2927 & 0,2922 \\
\hline
\end{tabular}

IV - MELHORIA DO FATOR DE POTÊNCIA ATRAVÉS DO AUMENTO DO NÚMERO DE PULSOS

Desprezando-se o efeito da comutação, tem-se que:

$F p=\left(\frac{\mathrm{I}_{1}}{\mathrm{I}}\right) \cos \alpha$

De acordo com a expressão (6):

$I=\sqrt{\sum_{n=1}^{\infty}\left(\frac{I_{1}}{n}\right)^{2}}$

Assim, para operação em 6 pulsos, tem-se:

$$
\mathrm{n}=6 \mathrm{k} \pm 1(1,5,7,11,13, \ldots)
$$

$\mathrm{Fp}_{6 \mathrm{p}}=0,9550 \cos \alpha$

Para 12 pulsos, tem-se:

$$
\begin{aligned}
\mathrm{n} & =12 \mathrm{k} \pm 1(1,11,13,23,25, \ldots) \\
\mathrm{Fp}_{12 \mathrm{p}} & =0,9901 \cos \alpha
\end{aligned}
$$

Para 24 pulsos, tem-se:

$$
\begin{aligned}
\mathrm{n} & =24 \mathrm{k} \pm 1(1,23,25,47,49, \ldots) \\
\mathrm{Fp}_{24 \mathrm{p}} & =0,9978 \cos \alpha
\end{aligned}
$$

Para 48 pulsos, tem-se:

$$
\begin{aligned}
\mathrm{n} & =48 \mathrm{k} \pm 1(1,47,49,95,97, \ldots) \\
\mathrm{Fp}_{48 \mathrm{p}} & =0,9996 \cos \alpha
\end{aligned}
$$




\section{V - CONVERSOR DE 12 PULSOS}

A. Um conversor de 12 pulsos convencional.

A figura 1 mostra um conversor de 12 pulsos convencional, formado por duas pontes graetz de 6 pulsos em série, alimentadas por transformadores conectados em estrela-estrela $\left(0^{\circ}\right)$ e estrela-delta $\left(30^{\circ}\right)$ - (Três enrolamentos).

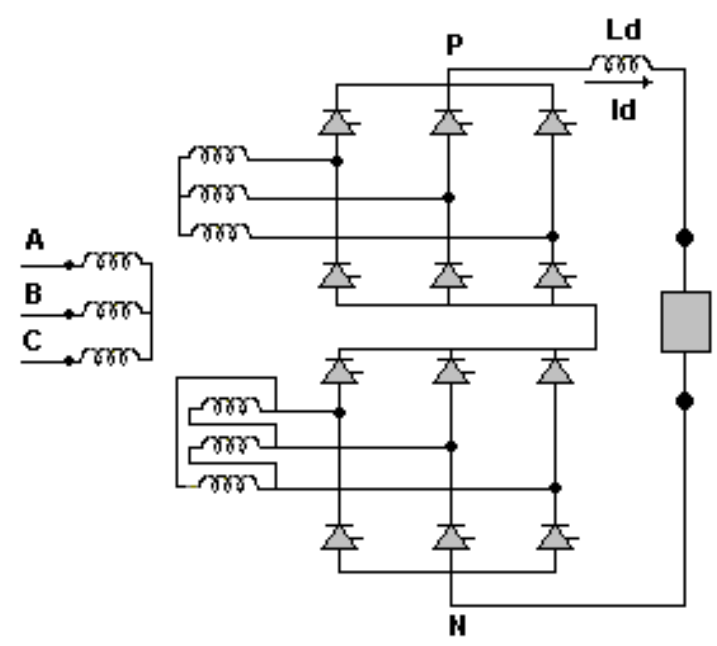

Figura 1- Conversor de 12 pulsos convencional.

Estes conversores são utilizados na maioria dos sistemas de conversão CA-CC (sistemas HVDC), como por exemplo, no projeto HVDC de ITAIPU.

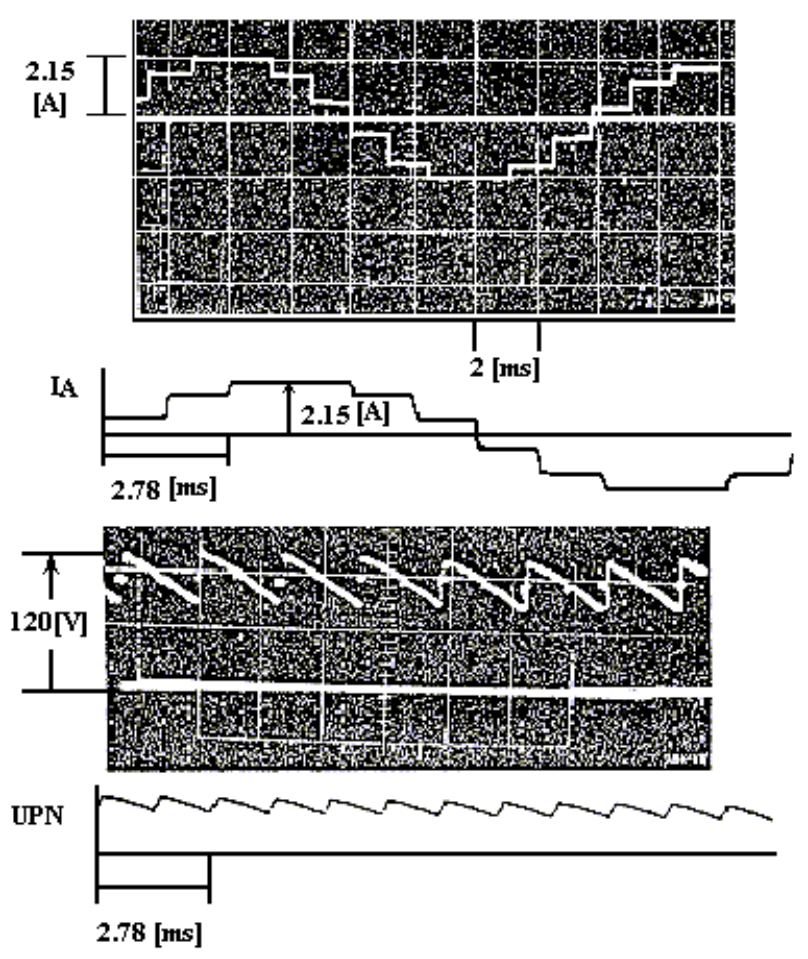

Figura 2-Formas de onda experimentais e simuladas de corrente de alimentação e tensão (CC) de saída para um conversor de 12 pulsos $\left(\alpha=30^{\circ} ; \mu=1,5^{\circ}\right)$.

A Figura 2 mostra as formas de onda experimentais e simuladas da corrente de alimentação e tensão (CC) de saída para ângulos de disparo e comutação de $\alpha=30^{\circ}$ e $\mu=1,5^{\circ}$, respectivamente.

\section{B. Conversor de 12 pulsos opcional [3].}

Uma diferente opção em 12 pulsos pode ser obtida através de transformadores com conexão estrela - delta estendido $\left( \pm 15^{\circ}\right)$. A Figura 3 mostra este tipo de transformador.

Os tapes ajustados para que se obtenha uma relação de transformação de 1:1 e uma defasagem angular de $\pm 15^{\circ}$ na tensão secundária são [1]:

$$
\begin{aligned}
& \mathrm{N}_{2}=0,518 \mathrm{~N}_{1} \\
& \mathrm{~N}_{3}=0,897 \mathrm{~N}_{1}
\end{aligned}
$$

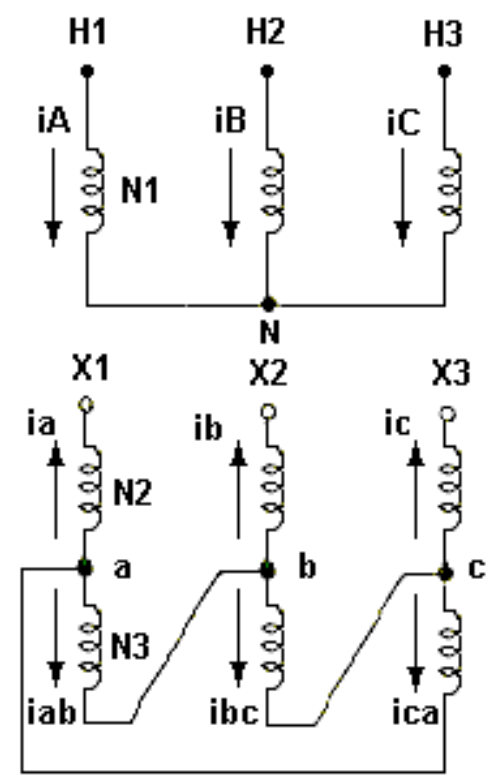

Figura 3 - Transformador com conexão estrela - delta estendido.

A Figura 4 mostra um conversor de 12 pulsos alternativo.

A Figura 5 mostra as formas de onda experimentais e simuladas da corrente de alimentação e da tensão (CC) de saída, para ângulos de disparo e comutação iguais a $\alpha=30^{\circ} \mathrm{e}$ $\mu=1,5^{\circ}$, respectivamente.

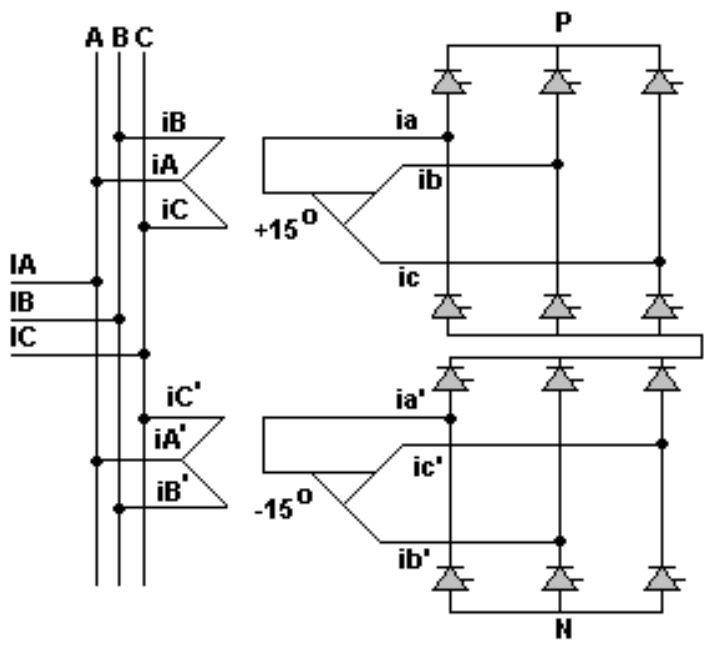

Figura 4 - Conversor de 12 pulsos alternativo. 

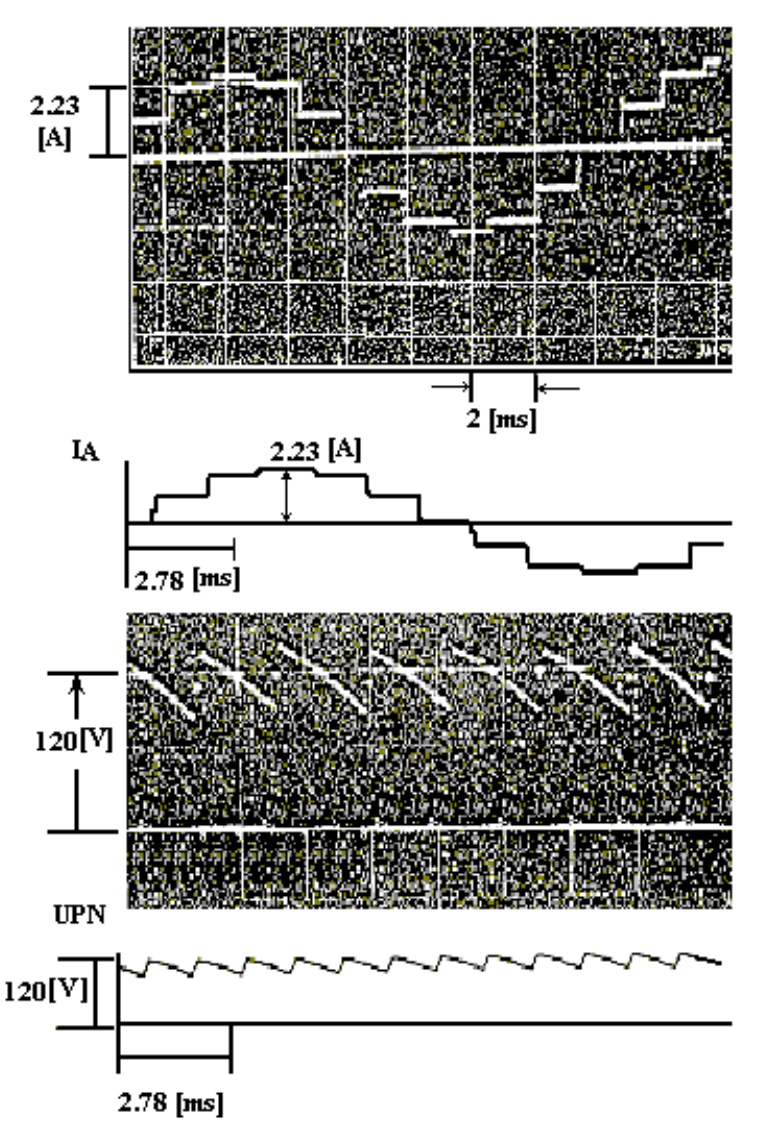

Figura 5 - Formas de onda experimentais e simuladas de corrente de alimentação e tensão $(\mathrm{CC})$ de saída para um conversor de 12 pulsos alternativo $\left(\alpha=30^{\circ} ; \mu=1,5^{\circ}\right)$.

\section{VI - CONVERSOR DE 24 PULSOS}

A. Conversor de 24-Pulsos utilizando transformadores conectados em delta zig-zag.

A Figura 6 mostra um transformador conectado em delta/zig-zag.

Os tapes $\mathrm{N}_{2}$ e $\mathrm{N}_{3}$ necessários para a obtenção dos defasamentos angulares: $-15^{\circ} ; 0^{\circ} ; 15^{\circ}$ e $30^{\circ}$ são apresentados na Tabela 2 [1].

TABELA 2

Tapes $\mathrm{N}_{2}$ e $\mathrm{N}_{3}$

\begin{tabular}{|c|c|c|}
\hline D.A. & TAPE N $_{2}$ & TAPE N $_{3}$ \\
\hline$-15^{\circ}$ & $141,42 \%$ & $51,76 \%$ \\
\hline $0^{\circ}$ & $100 \%$ & $100 \%$ \\
\hline $15^{\circ}$ & $51,76 \%$ & $141,42 \%$ \\
\hline $30^{\circ}$ & $0 \%$ & $173,21 \%$ \\
\hline
\end{tabular}

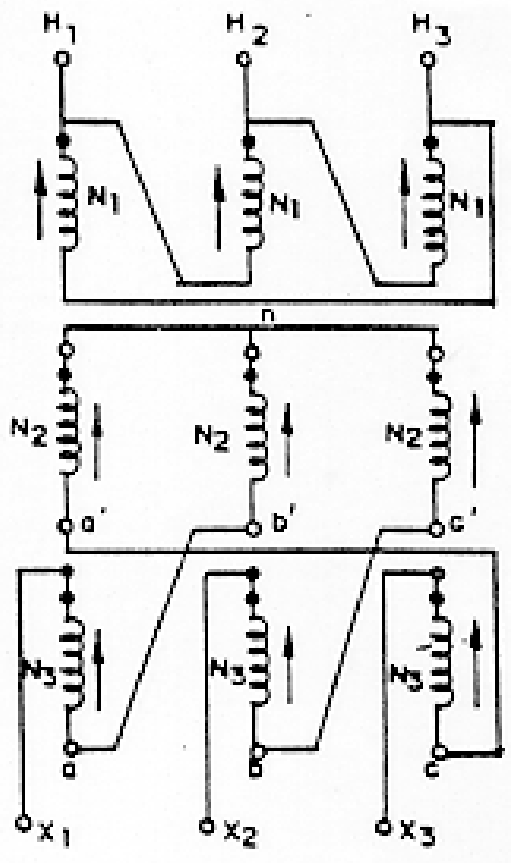

Figura 6 - Transformador conectado em Delta / Zig-Zag.

B. Conversores de 24 Pulsos alimentados por transformadores com conexões estrela-estrela $\left(0^{\circ}\right)$; estrela delta $\left(30^{\circ}\right)$; estrela - delta estendido $\left( \pm 15^{\circ}\right)$.

Estes sistemas de 24 pulsos podem ser obtidos através da associação convencional (figura 1) e alternativa (figura 4) de conversores de 12 pulsos. O defasamento angular de $15^{\circ}$ na tensão secundária é novamente alcançado.

\section{Resultados Experimentais e de Simulação}

A Figura 7 mostra um conversor de 24 pulsos, onde podem ser visualizadas as duas opções apresentadas anteriormente em VI A e VI B.

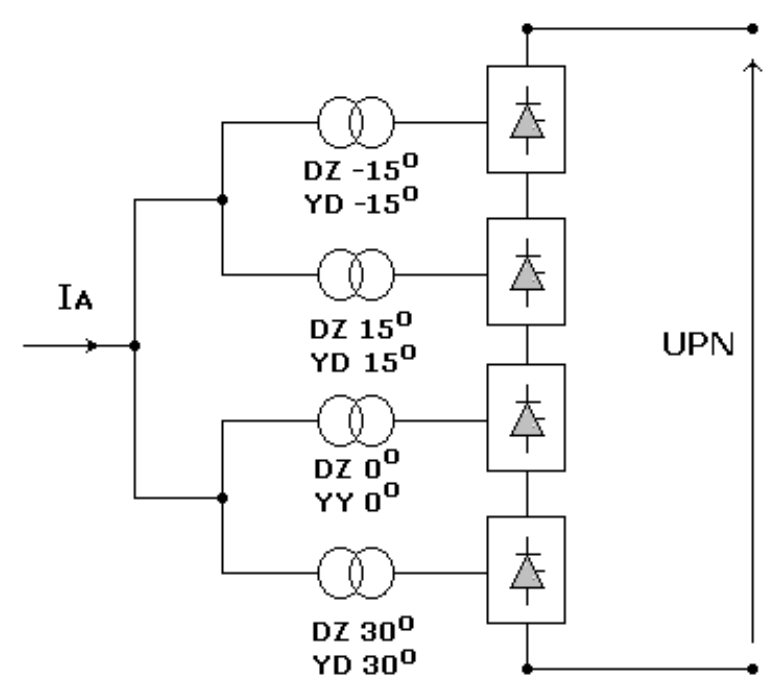

Figura 7 - Conversor de 24 pulsos. 


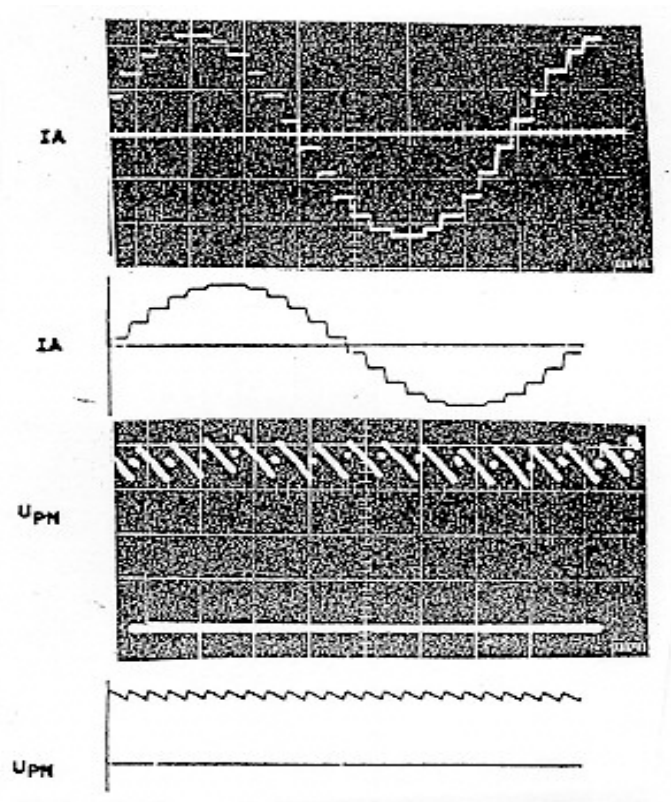

Figura 8 - Formas de onda experimentais e simuladas de corrente de alimentação $\left(\mathrm{I}_{\mathrm{A}}\right)$ e tensão CC de saída (UPN) para um conversor de 24 pulsos $\left(\alpha=30^{\circ} ; \mu=1,5^{\circ}\right)$.

Corrente: 1 div. Vertical $=2[\mathrm{~A}]$

Escala Horizontal: 1 div. $=2[\mathrm{~ms}]$

Tensão: 1 div. Vertical $=50[\mathrm{~V}]$

Escala Horizontal: 1 div. $=1[\mathrm{~ms}]$

A Figura 8 mostra as formas de onda experimentais e simuladas de corrente de alimentação (IA) e tensão $\mathrm{CC}$ de saída (UPN) para $\alpha=30^{\circ}$ e $\mu=1,5^{\circ}$.

\section{VII - CORRENTE CA EXPERIMENTAL PARA UM MULTICONVERSOR DE 48 PULSOS}

A Figura 9 mostra um multiconversor de 48 pulsos (configuração série).

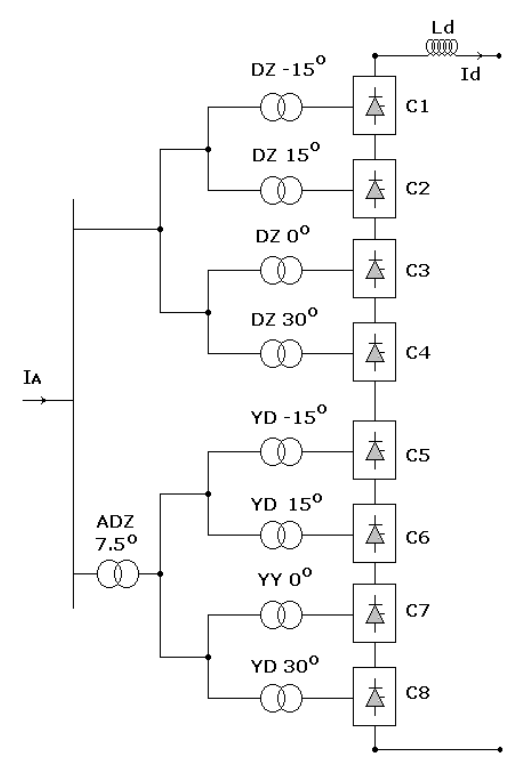

Figura 9 - Multiconversor de 48 pulsos (configuração série).
O equipamento ADZ mostrado na figura 9 é um autotransformador defasador $\left(7,5^{\circ}\right)$ [10]. Este equipamento possui relação de transformação $1: 1$, sendo responsável apenas pela introdução do defasamento necessário de $7,5^{\circ}$ nas tensões dos secundários dos transformadores alimentadores dos conversores.

A Figura 10 mostra o aspecto da corrente CA, IA e da tensão de fase $U_{\text {an }}$ para $\alpha=0^{\circ}$ e $\mu=4^{\circ}$ [4].

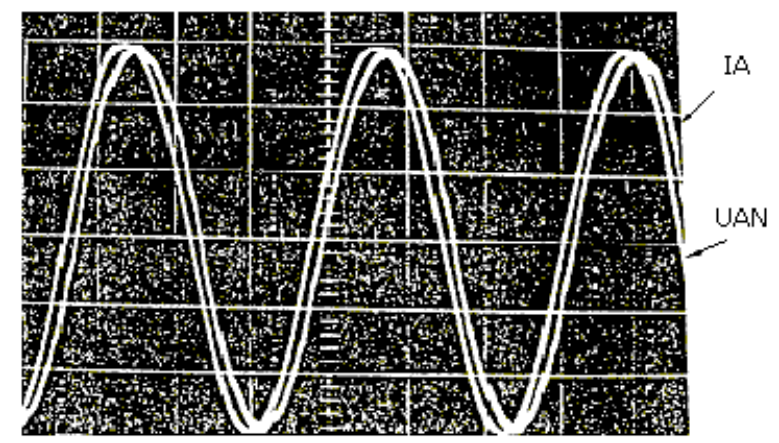

Figura 10 - Corrente $C A I_{a}$ e tensão de fase $U_{a n}$ de um multiconversor de 48 pulsos $\left(\alpha=0^{\circ} ; \mu=4^{\circ}\right)$.

Corrente: 1 div. Vertical $=3[\mathrm{~A}]$

Tensão: 1 div. Vertical $=60[\mathrm{~V}]$

Escala Horizontal: 1 div. $=5[\mathrm{~ms}]$

\section{VIII - RESULTADOS EXPERIMENTAIS}

Conversores de 6-12-24-48 pulsos formados por pontes de diodos conectadas em serie foram desenvolvidos em laboratório, a fim de se comparar os resultados teóricos de fator de potência calculados através das expressões $(15,16$, $17,18)$ com os resultados experimentais obtidos durante ensaios. Como pontes a diodos foram utilizadas, equivale a considerar o ângulo de disparo igual a zero graus nas expressões (15), (16), (17) e (18), para cálculo dos fatores de potência dos conversores.

Uma carga de resistência variável foi conectada aos terminais de saída (CC) para o multiconversor operando com 6,12 e 24 pulsos e considerando-se em todos os casos tensão e corrente de saída CC iguais a 220 [V] e 1,0 [A], respectivamente.

$\mathrm{O}$ equipamento de medição utilizado para medir o fator de potência durante os ensaios foi um medidor VOLTECH PM 300 (Three phase-power analyser), apropriado para medições em circuitos com formas de onda não senoidais.

Os resultados obtidos podem ser observados nas expressões 21 a 23 .

$\mathrm{Fp}_{6 \mathrm{p}}=0,9560$

$\mathrm{Fp}_{12 \mathrm{p}}=0,9920$

$\mathrm{Fp}_{24 \mathrm{p}}=0,9980$

Para outros tipos de carga, como cargas resistivasindutivas, os resultados obtidos foram praticamente os mesmos. Considerando-se um aumento da corrente da saída, 
o ângulo de comutação aumentará, acarretando numa maior aproximação da forma de onda da corrente de alimentação com uma senóide. Isto implicará no aumento do fator (FDH $=\mathrm{I}_{1} / \mathrm{I}$ ), o que a princípio possibilitaria um aumento do fator de potência. Entretanto, o ângulo de fase da componente fundamental da corrente, $\phi_{1}$, aumenta (vide equação 3) e o correspondente fator de potência diminuirá por este motivo (vide equação 1). Nas verificações experimentais feitas no artigo o ângulo de comutação era baixo e foi desprezado, e comparou-se, desta maneira os resultados calculados desprezando-se a comutação com os resultados experimentais, obtendo-se uma boa aproximação entre ambos. Para o caso de uma comutação não desprezível espera-se também uma aproximação entre resultados teóricos de fator de potência utilizando-se e equação 3 e os resultados experimentais.

\section{IX - CONCLUSÃO}

Os sistemas multiconversores estão sendo aplicados recentemente em novos sistemas elétricos, conforme pode ser comprovado pelas referências [16], [17].

Pode-se concluir que há uma melhoria no fator de potência de conversores quando se aumenta o número de pulsos deste conversor, devido ao aumento do Fator de Distorção de Harmônicos (FDH = $\left.\mathrm{I}_{1} / \mathrm{I}\right)$. Com FDH igual a 1 , equivale a dizer que a onda é puramente senoidal. À medida que ocorre diminuição de FDH, significa que a onda é mais rica em harmônicos. O programa FFT utilizado [5] apresentou uma boa precisão, como mostra a tabela 1 .

Transformadores conectados em estrela - delta estendido são uma boa opção para a implementação de um conversor de 12 pulsos, considerando-se que as unidades são idênticas, sendo que a simples inversão da seqüência de fases de uma delas é suficiente para a obtenção de uma defasagem angular $\pm 15^{\circ}$.

Através da associação de um conversor de 12 pulsos convencional com um conversor de 12 pulsos alternativo, pode-se obter um multiconversor de 24 pulsos. Este arranjo pode ser utilizado em sistemas industriais e também em sistemas HVDC de transmissão de energia elétrica, minimizando assim a instalação de filtros para harmônicos.

A comutação é responsável apenas pela atenuação de harmônicos, e não pela eliminação destes. Há na verdade, um aumento do fator de distorção de harmônicos (FDH), no entanto, o fator de potência do conversor diminui, porque o ângulo de fase $\phi_{1}$ da corrente fundamental aumenta (ver expressão 1).

A principal vantagem de se utilizar um autotransformador ADZ (figura 9), para a obtenção de uma defasagem angular de $7,5^{\circ}$ necessária para operação de um multiconversor de 48 pulsos, é o baixo custo deste tipo de equipamento.

Protótipos de multiconversores de 12-24-48 pulsos foram construídos e testados em nossos laboratórios, de acordo com $[1],[2],[4],[6],[9],[11],[12],[13],[14]$, [15] e os resultados experimentais obtidos (expressões 21, 22, 23) conferem com os resultados obtidos através de expressões teóricas para o cálculo do fator de potência de multiconversores (expressões 15, 16, 17). Pode-se assim afirmar que o valor do fator de potência experimental esperado para um conversor de 48 pulsos certamente estará próximo dos resultados teóricos mostrados na expressão 18 . As formas de ondas de correntes e tensões simuladas, também foram comprovadas pelos resultados experimentais obtidos (figuras 2,5,8), verificando-se desta forma a eficiência dos programas de simulação utilizados [11].

\section{REFERÊNCIAS BIBLIOGRÁFICAS}

[1] A.J.J.Rezek, "Modelagem e implementação de sistemas multiconversores", Tese de doutorado, FEE/UNICAMP, maio, 1991.

[2] A.J.J.Rezek, “Análise em regime permanente e transitório de um sistema de conversão de energia elétrica AC/DC" , Dissertação de mestrado, EFEI, Junho, 1986.

[3] L.Carlsson. "Extended-delta converter transformer for 12 - pulse operation in HVDC projects", International HVDC Seminar, Rio de Janeiro, Brasil, 1986.

[4] J.P.G.Abreu; A.J.J.Rezek; A.Candido, "Modelling and implementation of 48-pulse multiconverter", Proceedings IEEE-ICHPS, Bologna, Italy, pp 50-54, Setembro, 1994.

[5] J.C.G.Siqueira; G.H.F.Floriano, "Espectro harmônico de uma função periódica, utilizando transformada de Fourrier", EFEI/CPq/DET, Itajubá, Brasil, 1987.

[6] A.J.J.Rezek; J.P.G.Abreu; V.F.Silva; M.S.Miskulin,

“ Novas alternativas para transmissão HVDC ", Cigré, VI ERLAC, Foz do Iguaçu, PR, Brasil, Maio, 1995.

[7] E.Ulmann, "Power transmission by direct current", Springer-Verlag, Berlin, Heidelberg, New York, 1975.

[8] E.W.Kimbark, "Direct current transmission", Vol I, Wiley Interscience, New York, 1971.

[9] A.J.J.Rezek; J.P.G.Abreu; V.F.Silva; M.S.Miskulin, "Aumento do número de pulsos do sistema conversor: conteúdo harmônico e melhoria do fator de potência", Cigré, VII ERLAC, Puerto Iguazú, Argentina, maio, 1997.

[10] J.P.G.Abreu; A.J.J.Rezek; V.F.Silva; L.E.B.Silva; M.S.Miskulin, "Harmonics elimination in multiconverter systems by using a special autotransformer (ADZ)" Proceedings AMSE Modelling, Simulation \& Control, AMSE Press, vol. 38, n 4, pp 45-53, France, 1992.

[11] A.J.J.Rezek; M.S.Miskulin; J.P.G.Abreu "Projeto auxiliado por computador aplicado a eletrônica de potência", II Seminário de Eletrônica de Potência, pp. 102-109, UFSC, Florianópolis, SC, Brasil, 1989.

[12] A.J.J.Rezek; J.P.G.Abreu; V.F.Silva; M.S.Miskulin, "Converters for application in HVDC systems", International Conference on Contribution of Cognition to Modeling, AMSE, July 6-8, Lyon-Villeurbanne, France, 1998.

[13] O.H.S.Vicentini; A.J.J.Rezek; "Melhoria do fator de potência e do conteúdo harmônico de conversores comutados pela rede", CNPq, Pesquisa de iniciação científica, 2000.

[14] A.J.J. Rezek; J.P.G.Abreu; V. F. Silva; J.M.E. Vicente; J.A. Cortez; W. O. Assis; M. S. Miskulin " Power factor improvement of line-commutated graetz converters by increasing their number of pulses", $V$ Congresso Brasileiro de Eletrônica de Potência, COBEP 99, Foz do Iguaçu, PR, Brasil, Anais COBEP'99, v.2. pp 551-556, 1999. 
[15] A. J. J. Rezek; J. P. G. de Abreu; V. F.da Silva; J. M. E. Vicente; J. A. Cortez; ${ }^{\circ}$ H. S. Vicentini; A. C. de Sá; M. S. Miskulin, "Power factor improvement of line-commutated graetz converters by increasing their number of pulses: modeling and experimental results" , artigo aprovado para publicação e apresentação no IEEE X ICHQP (International Conference on Harmonics and Quality of Power), de 6 a 9 de Outubro de 2002, Rio de Janeiro, Brasil.

[16] S. Mori; K. Matsuno; T. Hasegawa; S. Onnishi; M. Takeda; M. Seto; S. Murakami; F. Isniguro, "Development of a large Static Var Generator using self-commutated inverters for improving power system stability", IEEE Transactions on Power Systems, vol. 8, N.1, pp. 371-377, february 1993.

[17] E. Cengelci; P. Enjeti; W. Gray “ A new modular motor-modular inverter (MM-MI) concept for medium voltage adjustable speed drive systems", Proceedings IEEE PESC, pp. 1972-1979, 1999.

\section{DADOS BIOGRÁFICOS}

Angelo José J. Rezek, nascido em 1959 em Conceição do Rio Verde (MG), é engenheiro eletricista (1981), mestre (1986) em engenharia elétrica pela Escola Federal de Engenharia de Itajubá - EFEI, e doutor (1991) em engenharia elétrica pela Universidade de Campinas - UNICAMP.É docente na atual UNIFEI desde 1981. Suas áreas de interesse são eletrônica de potência e máquinas elétricas.

José Policarpo Gonçalves de Abreu, nascido em 1952 na Ilha da Madeira, Portugal, é engenheiro eletricista (1975), mestre (1980) em engenharia elétrica pela Escola Federal de Engenharia de Itajubá - EFEI, e doutor (1991) em engenharia elétrica pela Universidade de Campinas UNICAMP. É docente na atual UNIFEI desde 1976. Suas áreas de interesse são aplicações de transformadores conversores, máquinas elétricas, e qualidade de energia elétrica.

José Antonio Cortez, nascido em 1953 em Itajubá (MG), é engenheiro eletricista (1979) pelo Instituto Nacional de Telecomunicações - INATEL, mestre (1987) pela Universidade de São Paulo (USP-São Carlos) e doutor (1997) em engenharia elétrica pela Universidade de São Paulo (USP-São Paulo). Suas áreas de interesse são eletrônica de potência e acionamentos elétricos.
Valberto Ferreira da Silva, nascido em 1951 em Bento Gonçalves (RS), é engenheiro eletricista (1976) e mestre (1980) em engenharia elétrica pela Escola Federal de Engenharia de Itajubá - EFEI, e doutor (2001) pela Escola Politécnica da Universidade de São Paulo. Suas áreas de interesse são eletrônica de potência e acionamentos elétricos.

José Manuel Esteves Vicente, nascido em 1960 em Fundão, Portugal, é engenheiro eletricista (1987) e mestre (1981) em engenharia elétrica pela Escola Federal de Engenharia de Itajubá - EFEI. Suas áreas de interesse são ensaios em máquinas elétricas e eletrônica digital.

Adriana Cortez de Sá, nascida em 1975 em São Paulo (SP), é engenheira eletricista (1998) pelo Instituto Nacional de Telecomunicações - INATEL 1998. É mestre em ciências em engenharia elétrica, título obtido em 2002 na Escola Federal de Engenharia de Itajubá (EFEI). Atualmente ela está cursando pós-graduação (doutorado) na área de eletrônica de potência da Universidade Federal de Itajubá - UNIFEI.

Otavio Henrique Salvi Vicentini, nascido em 1979 em Itapira (SP), graduou-se em agosto de 2002 em engenharia elétrica pela Universidade Federal de Itajubá (UNIFEI). Suas áreas de interesse são máquinas elétricas e eletrônica de potência.

Mauro Sérgio Miskulin, nascido em 1947 em Santa Rita do Passa Quatro (SP), é engenheiro eletricista (1971), mestre (1974) em engenharia elétrica pela Universidade de Campinas - UNICAMP, e doutor (1980) em engenharia elétrica por Cranfield Institute of Technology, Inglaterra. Suas áreas de interesse são eletrônica de potência e máquinas elétricas.

Adriana Aparecida dos Santos Izidoro, nascida em 1973, em Cristina (MG), é engenheira eletricista formada em 1995 pelo Instituto Nacional de Telecomunicações (INATEL), mestre em ciências em engenharia elétrica pela Escola Federal de Engenharia de Itajubá (EFEI), 2000. Atualmente ela está cursando pós-graduação (doutorado) na área de eletrônica de potência da Universidade Federal de Itajubá UNIFEI. 